\title{
Behavioural modernity, investigative disintegration \& Rubicon expectation
}

\author{
Andra Meneganzin $^{1}$ (D) Adrian Currie ${ }^{2}$ (D)
}

Received: 1 February 2021 / Accepted: 7 November 2021 / Published online: 26 February 2022

(c) The Author(s) 2022

\begin{abstract}
'Behavioural modernity' isn't what it used to be. Once conceived as an integrated package of traits demarcated by a clear archaeological signal in a specific time and place, it is now disparate, archaeologically equivocal, and temporally and spatially spread. In this paper we trace behavioural modernity's empirical and theoretical developments over the last three decades, as surprising discoveries in the material record, as well the reappraisal of old evidence, drove increasingly sophisticated demographic, social and cultural models of behavioural modernity. We argue, however, that some approaches to identifying and categorizing modernity have not kept up with this new picture. This is due to what we term 'Rubicon expectations': classificatory and interpretive practices which look for or assume clear demarcations in behavioural and cultural processes. We develop a philosophical account of 'investigative disintegration' to capture how our understanding of behavioural modernity has changed, and how Rubicon-based practices have become inadequate. Disintegration, in the form we analyse, occurs when scientists' conception of a phenomenon shifts sufficiently to reshape an investigation's epistemic structure. For behavioural modernity, the explanatory weight which once lay on identifying 'switch-points' in the innate suite of hominin cognitive capacities, lies now in understanding the social and demographic environments that were capable of sustaining and nourishing more complex material cultures. Finally, we argue that the phenomenon itself has not disintegrated to the point that we are left with no interesting explanandum: for all its mosaic, disparate nature, there are still good reasons for behavioural modernity to retain its central place in investigation of our species' origins.
\end{abstract}

Andra Meneganzin

andrashirin.meneganzinabwini@phd.unipd.it

Adrian Currie

a.currie@exeter.ac.uk

1 Department of Biology, Evolutionary Biology Research Unit, University of Padua, Via U. Bassi 58/B, Padova, Italy

2 Department of Sociology, Anthropology and Philosophy, University of Exeter, Byrne House, Stocker Road, Exeter EX4 4PY, UK 
Keywords Archaeology - Behavioural modernity - Cumulative cultural evolution · Homo sapiens · Investigative disintegration · Rubicon expectation

\section{Abbreviations}

AMHs Anatomically Modern Humans

LSA Later Stone Age

MSA Middle Stone Age

MP-UP Middle to Upper Palaeolithic transition

OOA Out of Africa

UP Upper Palaeolithic

\section{Introduction}

Generalist accounts of science's structure and progress have failed: science is a far too heterogeneous, contingent and human beast for universally applied, abstract schema to gain traction. In light of this, philosophers are developing local, context-sensitive and pragmatic models of science. Reduction is replaced with mechanistic understanding (Machamer et al., 2000), unity with local integration, disunity with a patchwork of independent and interdependent representations, epistemic goods and tools (Brigandt, 2010; Mitchell, 2003; Potochnik, 2010; Wylie, 1999). Much work in this vein has attended to the formation and stability of research; how local integration and independence between evidence, theories and institutions structure science and its epistemic standing (Ankeny \& Leonelli, 2016; Chang, 2012). However, progress in scientific investigation isn't solely attained via integration, that is, aligning new data into stable theoretical horizons, research strategies and agendas. New data and new re-readings of available data may also disrupt fundamental research questions, approaches and concepts. In this paper we examine such disruption: investigative disintegration. How new, unexpected results reshape our conceptions of target phenomena, thus reshaping epistemic landscapes.

We'll examine disintegration through a close look at developments in the study of our species' behavioural origins. 'Behavioural modernity' traditionally indicates distinctively human behavioural and cultural expressions. The notion was initially introduced to mark the perceived time-lag between the apparent arisal of $\mathrm{H}$. sapiens-anatomical modernity-now set at ca. 300-200kya (Hublin et al., 2017; Lipson et al., 2020; McDougall et al., 2005; Schlebusch et al., 2017), and the stabilization of the archaeological assemblage associated with characteristic human behavioural and cognitive capacities, such as sophisticated sociality or symbolic expression, after ca. 100-50 kya (Henshilwood \& Marean, 2003; Renfrew, 1996, 2009). The temporal mismatch was thought by archaeologist Colin Renfrew to pose a genuine explanatory puzzle - the so-called "sapient paradox": why did it take more than 100.000 years for the modern body to meet the modern mind? 
Over twenty years, a richer set of archaeological and paleontological specimens, as well as genomic data from Africa and globally, provided richer contextual information about population and species distribution, migration and interaction, and more sophisticated accounts of the factors underlying material complexity (see Galway-Witham et al., 2019 for a review). As we'll describe in Sect. 2, what counts as a relevant archaeological signal in this context has been progressively rethought and the evolutionary models explaining such signals have been complexified, thus significantly reshaping our conception of behavioural modernity.

Today, the nonlinear and divergent ways in which traits of behavioural modernity appeared in various regional contexts are ever-more emphasized features of the archaeological record. Recent research has increasingly moved away from saltationist and hardwired causal approaches, making older classificatory and interpretive strategies no longer on par with current epistemic standards, yet some of these are retained. In Sect. 3 we highlight one example of this—Rubicon expectation-where archaeological investigation and categorization are expected to provision a kind of 'switch-point' marking where and when the phenomenon arose. For behavioural modernity, this involves identifying discrete and unambiguous cognitive or behavioural boundaries that should divide early and late anatomically modern humans (AMHs), ${ }^{1}$ or biologically distinct hominin species.

To capture these crucial features in the evolution of the research surrounding behavioural modernity, in Sect. 4 we will develop an account of 'investigative disintegration'. Investigations disintegrate when a once-clear phenomenon becomes complexified such that the epistemic structure of the investigation transforms. Disintegration, we'll show, doesn't simply involve changes to empirical and theoretical regimes but shifts in explanatory weight. Previously, the explanatory weight on accounts of behavioural modernity fell on explaining when and how distinctive cognitive and behavioural clusters of traits arose. In the new context the explanatory weight falls on the conditions by which such traits became sufficiently stabilized at a regional scale for cumulative cultural evolution. We'll argue that recent discoveries and new interpretive perspectives have changed the explanatory game such that 'Rubicon'-style explanations and approaches to categorization are not adequate for the current research agenda, and thus cannot carry the explanatory weight required for understanding the phenomenon. We'll argue that in light of investigative disintegration the significance of archaeological discoveries regarding modernity should shift, but that despite all this, the concept of 'behavioural modernity' should be retained.

Our paper, then, is intended both to provide a philosophical analysis of 'investigative disintegration', which we suspect is a not-infrequent scientific phenomenon, and to apply our analysis to the evolving research agenda targeting behavioural modernity. We'll do this in two major steps. The first, Sects. 2 and 3, will summarize and critique research on behavioural modernity. The second, comprising Sects. 4 and 5, will shift to more abstract philosophical analysis, analysing investigative disintegration and bringing it to bear on behavioural modernity.

\footnotetext{
1 Although the usage of the term "anatomically modern humans" may vary in scope, it was introduced to distinguish hominin fossils that fall within the range of today's human skeletal variation from otherwise anatomically "archaic" forms.
} 


\section{Behavioural modernity in hindsight}

How 'behavioural modernity' is understood, as well as the proposed mechanisms underlying its emergence, have been radically transformed (d'Errico \& Stringer, 2011; Davies, 2019). In this section, we'll discuss how the relationship between the archaeological record and the phenomenon at hand has shifted as new data has come online, before tracing the new explanatory models that have been produced in light of this.

\subsection{Signals of modernity}

From an archaeological perspective, 'behavioural modernity' can be understood as a phenomenon inferred from the record based on a constellation of material proxies taken to be revelatory of complex cognitive, technical and social lives. Debate, then, often turns on the appearance and distribution of such proxies (Henshilwood \& Marean, 2003). These traditionally took the form of 'trait-lists': an inventory of discrete detectable material signatures taken to provide universal indicators of modern behaviour. Common examples are new lithic technologies (blades and microblades), the appearance of composite tools; worked bone, antler and ivory; long distance exchange of raw materials; seasonal mobility and exploitation of resources; elaborate and varied art forms such as engravings, sculptures and cave paintings; musical instruments; widespread use of personal ornaments; manipulation of symbol and notation systems (cfr. Ames et al., 2013; Henshilwood \& Marean, 2003; McBrearty \& Brooks, 2000).

Such trait-lists were initially based on Upper Palaeolithic European sites (henceforth UP; Gamble, 1994; Mellars \& Stringer, 1989). Through the noughties lists more reflective of the African Middle Stone Age (MSA) were developed in an attempt to correct this Eurocentric bias (Deacon, 2001; McBrearty \& Brooks, 2000). Critically, signatures were no longer treated as a package deal or a 'syndrome', instead they were taken to reflect scattered origins in space and time. Lately, research has targeted particular aspects of material culture thought to provide clear signals of behavioural modernity: what we call archaeological "golden spikes". These focus especially on symbolic expression tied to, for instance, pigment processing (e.g., ochre) or bead production (Henshilwood \& Marean, 2003; Marean, 2015; Tattersall, 2008; Wadley, 2001).

It now seems like an obvious mistake to use the European UP record (ca. 40-10 kya) as an empirical yardstick to establish contrasts between late and early modern Homo sapiens in Africa (D'Errico, 2003; Deacon, 2001; Henshilwood \& Marean, 2003; Shea, 2011). However, some have argued that recognising the limitations and European origin of such working definitions has neither led to complete abandonment nor to replacement with substantially different approaches (Barham \& Mitchell, 2008). Employing the record of any single region as a standard for judging global patterns in human evolution risks conflating regional dynamics and universal trends (especially if fieldwork has been historically prioritized in that region, Gamble, 1999; Shea, 2011). To see this, let's trace some influential developments in how modernity has been detected in the archaeological record. 
Against Eurocentrism, McBrearty and Brooks (2000) developed a trait list aimed at better reflecting the depth and complexity of the African archaeological record, thus showing that many purported European innovations had African precursors. They proposed four sets of traits characterizing modern behaviour: abstract thinking; planning depth; behavioural, technological and economic innovativeness and-notably-symbolic behaviour. These were linked with numerous archaeological signatures which were taken to demonstrate their acquisition. As we'll see in 2.2, the overall model behind the appearance of such traits differs substantially from those developed based on the UP European package. However, the list, as D'Errico (2003) rightly noted, lacked cross-cultural comparative analysis of various human societies and was still derived from local material culture (African MSA and, to a lesser extent, the UP). This, he argued, provides poor heuristics for testing modern characteristics of populations living in different environments which might have followed different evolutionary trajectories.

Henshilwood and Marean (2003) identified four major flaws in defining behavioural modernity through trait-lists (or, as Wadley, 2001 ironically called them, "shopping lists"): empirical derivation, ambiguity, lack of theoretical grounding and taphonomic bias. ${ }^{2}$ Although these objections first applied to European-derived traits, they are plausibly applicable to any attempt to approach modern behaviour through trait-lists developed from particular sites and particular time periods as opposed to world-wide anthropological and ethnographic data (see also Wynn et al., 2016). So, deriving markers from geographically localized archaeological records (be they African MSA or European UP) makes behavioural modernity unambiguously recognizable only in the records on which the list was based (D'Errico \& Banks, 2013). Further, proxies taken to signal newly evolved capacities may instead be reactions to resource intensification or population pressure (hence, they are ambiguous because other processes can be invoked to explain their appearance in the record). Moreover, the inclusion of some traits in the lists seems to lack proper theoretical justification: for example, seasonal mobility, besides varying widely among living humans, is known to be practiced by some non-human species as well (Wadley, 2001). Finally, as with many archaeological investigations, taphonomic biases are a constant worry, especially in equatorial contexts where biotic preservation (e.g. bone or antler tools) is unlikely.

Having critiqued trait-lists as being inherently flawed, Henshilwood and Marean conclude that a better approach would be to instead focus on one particular proxy that gathers consensus for being a crucial modern human feature: symbolic expression and its material signatures. Frequently cited are data signalling symbolic capacities from South African sites, such as mineral pigment modification (ochre) and perforated shells found at Blombos Cave (100-70 kya), later reinforced by new finds and revised dates at other sites (see Wadley, 2015 for an updated review). This all led to a growing focus on a "golden spike" for modernity: the entry into the record of materials thought to unambiguously signal symbolism. These, as we'll show, are in fact equally susceptible to the flaws identified for trait-lists. In Sect. 4 we'll argue that both trait-lists

\footnotetext{
2 Taphonomic bias refers here to the differential preservation in the archaeological record of the traits used to diagnose behavioural modernity. This can likely lead to false negative findings about modern behaviour in regions where the potential for preservation is generally low.
} 
and 'golden-spikes' are problematic when used as Rubicons, in light of investigative disintegration.

Another thread running alongside debates about proxies asks whether behavioural modernity can (or should) identify a unique set of behaviours characterizing AMHs and not other hominin species. Where previously behavioural modernity was meant to distinguish our species from Neanderthal proxies, as Zilhao provocatively noted these often ended up "defining some modern humans as behaviourally Neandertal and some Neandertal groups as behaviourally modern" (2006). As archaeological signals of behavioural modernity became increasingly diffuse, identifying the phenomenon as a particular process restricted to a single lineage became less plausible, especially in light of parallel arguments stressing the cognitive and social sophistication of our non-sapiens cousins (e.g., Stiner 2017; Villa \& Roebroeks, 2014). However, some still object that complex traits such as imaginative and symbolic intelligence were expressed less systematically in Neanderthals (Tattersall, 2017).

Problems of definition regarding what exactly is meant by the term 'behavioural modernity' persist (see Nowell, 2010), but elaborating definitory alternatives is outside our scope (we'll likely end up reproducing old problems in new forms). ${ }^{3}$ We'll continue referring to 'behavioural modernity' with the purpose of highlighting the various investigative dimensions along which research has 'disintegrated' (see Sect. 4), stressing available and more promising ways of rethinking the problem at hand. This disintegration of our conceptions of the phenomenon of behavioural modernity has led to the generation of new explanatory models, which we'll turn to now.

\subsection{Models of modernity}

Alongside asking how behavioural modernity should be detected in the archaeological record-how to characterize the phenomenon at hand-researchers have developed various models for the evolution of behaviourally modern characteristics in hominin populations. Such models differ with regards to the evolutionary trajectory of behavioural change, the geographic focus, and timing and relevant causal factors (see Table 1 below).

Here are summarized six main models that are reviewed in the paper (though more have been proposed). Although overlaps and similarities are clearly possible, the distinctions are useful for detecting relevant differences in one or more of the following features: trajectory, timing, geographical focus and range of causal factors.

In the 80's and 90's, the basic picture of how and when our hominin ancestors became like us was commonly understood. According to the "Upper Paleolithic model", the path leading to the modern mind was abrupt and exclusively associated with AMHs and their arrival in Europe (Bar-Yosef, 1998, 2002; Diamond, 1992; Klein, 1989a, 1989b; Mellars \& Stringer, 1989). The archaeological record of the UP, exhibiting a proliferation of new, successful technological and cultural changes suggested a "lightbulb moment" in human evolution. Early trait-list approaches on which the revolutionary scenario was based tended to suggest that innovations arrived as a 'package'

\footnotetext{
${ }^{3}$ As Kim Sterelny suggests, defining behavioural modernity as falling within the range of variation of ethnographically known foragers is reasonable and neutral (personal communication).
} 


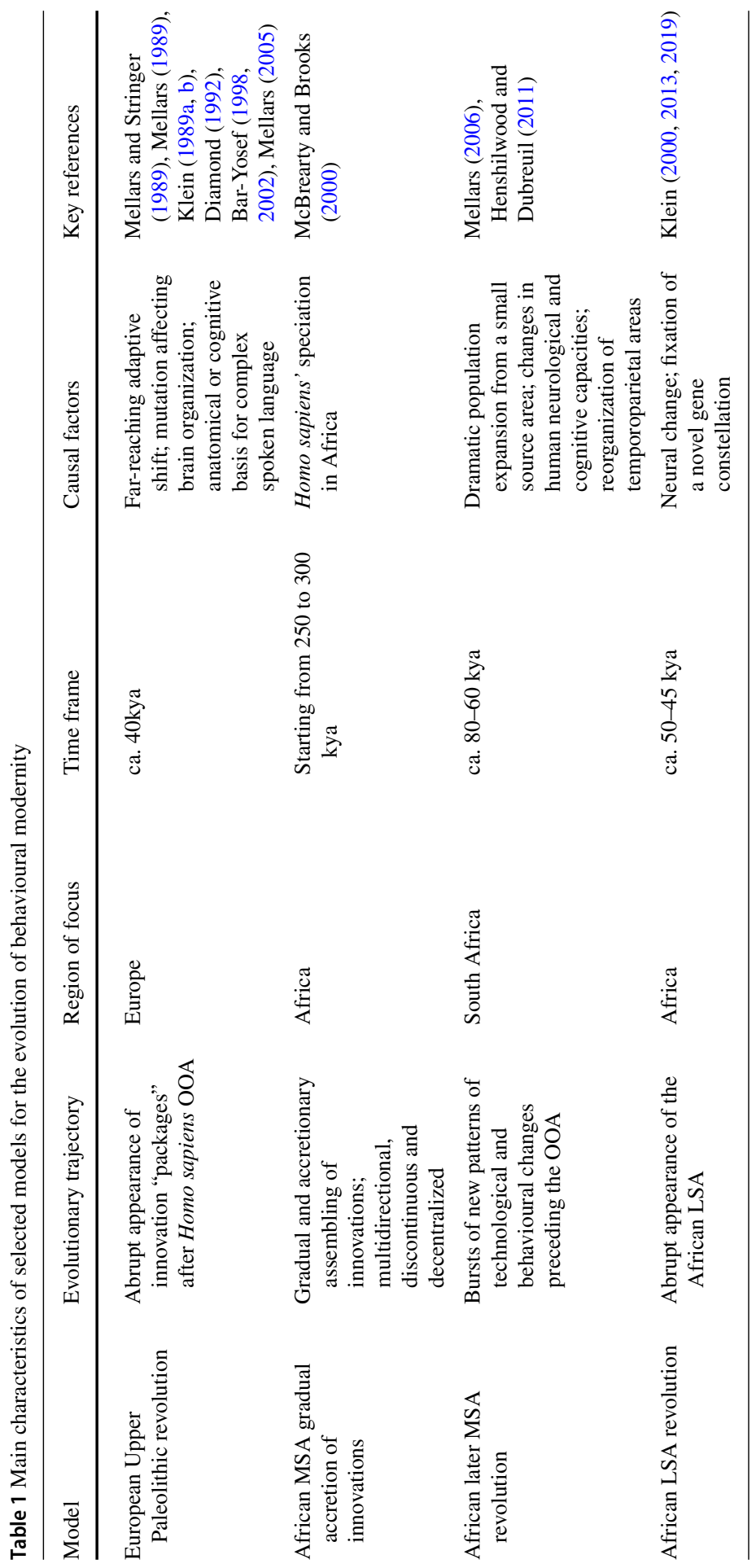




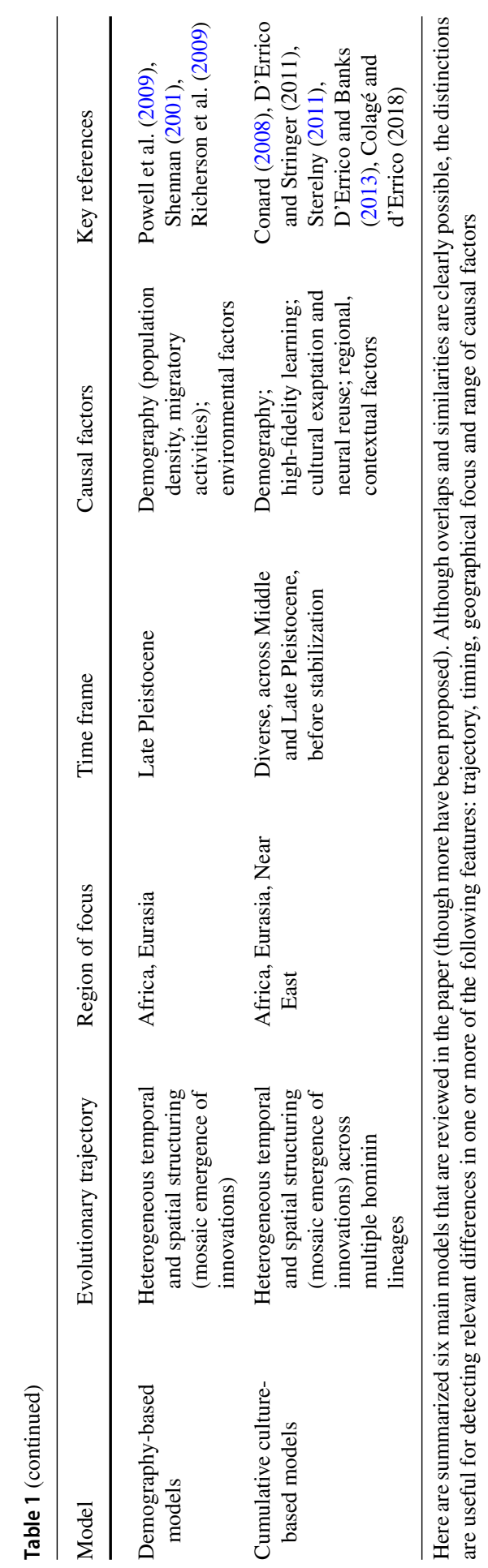


during the Middle to Upper Palaeolithic transition (MP-UP). This inflection in the record was interpreted not as a biased discontinuity but as reflecting a major cognitive and behavioural breakthrough, a "creative explosion" (Mithen, 1998; Renfrew, 2009) so marked that some scholars argued it was probably caused by a selectively advantageous genetic mutation, occurring around 50-40 kya, affecting brain functioning and internal organization (Klein, 1989a, 1989b). Others thought of changes in the anatomical or cognitive basis for spoken complex language as a plausible answer (e.g. Diamond, 1992; Mellars, 1996).

Within this perspective, there is an "impossible coincidence" (Mellars, 2005) between Homo sapiens' range expansion across the European continent (following the so-called Out-of-Africa, OOA, dated at ca. 70-60 kya) and the almost simultaneous emergence throughout the Old World of technological and cultural innovations marking MP-UP transition. Resolving the coincidence led to a "single-species model" for the origin of modern behaviour, which excluded the convergent or parallel evolution of cognitive sophistication in other archaic hominin lineages (notably Neanderthals), thus accounting for the different evolutionary trajectories followed by "invaders" sapiens and "indigenous" Neanderthal populations, the former replacing the latter (ivi; see Villa \& Roebroeks, 2014 for criticism).

However, as we've seen in Sect. 2.1, a growing body of archaeological evidence supports the emergence of key cultural innovations in Africa before the purported European creative explosion. A wealth of counterevidence has accumulated against the "revolution" scenario, that many take to favour a more gradual evolutionary picture.

According to McBrearty and Brook's explanatory model, modern behaviour must have been the consequence of Homo sapiens' speciation in Africa. Therefore, a gradual accretion of innovations should be visible in the archaeological record as a result of the selective pressures leading to the emergence of AMHs (Marean et al., 2007; McBrearty \& Brooks, 2000). The time-lag between the origin of anatomical modernity and signals of behavioural modernity is considerably reduced within the gradualist perspective and associated with the onset of the African MSA. In explaining the expansion from Africa no genetically encoded, dramatic change is invoked: traits characterizing modern human behaviour were developed by hominin groups through cognitive and behavioural capabilities that were plausibly already in place. However, it has also been argued that the accretionary pattern could be a methodological artifact, due to mixing traits belonging to the African MSA and the European UP (D'Errico \& Banks, 2013; Shea, 2011).

Rather than abandoning the idea of a breakthrough, some researchers placed it earlier and in Africa. For instance, Mellars (2006) has proposed that this moment should be located between 60 and 80 kya in southern Africa, concomitantly with a major population expansion and with the appearance of technological and behavioural innovations in the Still Bay and Howiesons Poort industries that, he argues, sharply contrast with those of earlier African MSA sites (see also Henshilwood \& Dubreuil, 2011). Although invoking adaptive environmental processes as a parsimonious (and more prosaic) explanation, Mellars nonetheless argues that significant changes in human neurological and cognitive capacities can in no way be ruled out. Henshilwood and Dubreuil (2011) invoke a reorganization in modern H. sapiens of the temporoparietal areas implicated in higher theory of mind. Klein $(2000,2013,2019)$ explicitly ties 
behavioural change to a fortuitous mutation or a novel gene constellation promoting the fully modern brain and the onset of the African Later Stone Age (LSA, 50-45 kya).

In parallel, however, alternative models have gained increasing consensus, radically reshaping the relevant questions and tackling different aspects of behavioural modernity. For example, the "demographic perspective" asks not how novelties emerge, but how cultural complexity is maintained (or lost) through time. Some scholars have argued that demography is a major determinant here, and that population size, density and migration activities, probably triggered by climatic and environmental dynamics, shaped the spatial structuring of cultural traits' accumulation and evolution (Powell et al., 2009; Richerson et al., 2009; Shennan, 2001), thus accounting for the asynchronous appearance, disappearance and re-emergence of key innovations within the African MSA.

Others have highlighted that such demographic-based mechanisms, far from being the unique explanatory factor in the spread and maintenance of innovation (see Sterelny, 2011 for the role of high-fidelity learning settings), are equally applicable to archaic hominins like Neanderthals, suggesting that their different evolutionary fate might have well been dependent on group size and rates of cultural exchange, rather than hardwired, "in-built" cognitive differences (d'Errico \& Banks, 2013; d'Errico \& Henshilwood, 2011).

Partisans of this last view, dubbed the "cultural model" (d'Errico \& Stringer, 2011), expand on the demographic perspective, and argue that modernity is the result of cumulative cultural evolution among populations that already had the capacity to be cognitively modern irrespective of taxonomic affiliation. Much of the cognitive prerequisites and cerebral hardware of modern behaviour were perhaps already present among the last common ancestors of Neanderthals and modern humans.

On such views, what explains the appearance, diffusion and acceptance of innovative behaviours-besides population pressures and demographic dynamics-is the ability of a society to create stable and high-fidelity learning settings within a specific, ecological and environmental context and given peculiar historical contingencies (D'Errico, 2003; d'Errico \& Banks, 2013; Hovers \& Belfer-Cohen, 2006; Zilhao, 2007). Here a multiple species model for the origin of behavioural modernity is invoked, contrasting the idea of a single speciation event as a main trigger. Modern traits appeared among different hominin groups and in different African and Eurasian regions, and the process of change (especially in the early phases of the MSA) was not linear nor progressive in nature, but rather followed a "mosaic" mode of evolution (see Parravicini \& Pievani, 2019). Here, innovations and traits appear and disappear (and reappear again in quite different forms, e.g. beads, see d'Errico et al., 2009) in an haphazard and irregular fashion, before becoming fully crystallized into a cohesive package. As for Europe, D'Errico (2003) does not exclude that contact among AMH and Neanderthals might have boosted innovation and the production of symbolic objects on both sides. What emerges then is an intricate and pluralistic picture, in which modern behaviour manifests itself as the result of multiple evolutionary trajectories followed by its constitutive traits, crosscutting hominin phylogeny. 
Although this is not the only scenario available in today's approach to human cultural and cognitive evolution, ${ }^{4}$ sociocultural and demographic based models (also aided by incorporation of elements of the Evolutionary Extended Synthesis, see Kissel \& Fuentes, 2021) are redrawing the conceptual toolkit and investigation. Further, it is argued that such scenarios are more grounded in the available evidence compared to biologically based explanations, as they account for patterns in the record without resorting to events that are not yet empirically sustained (nor easily testable). For instance, it is well-established that major population growth took place in Africa before the radiation into Eurasia, likely favouring cultural accumulation (Henn et al., 2012), whereas as of today no species-wide genetic sweep just before 50 kya has been found (Mallick et al., 2016).

It is clear that the notion of 'behavioural modernity' (and the whole research agenda around it, as we shall see) has undergone significant change. The causal link between modern anatomy and modern behaviour has been gradually loosened (along with the one human taxon/one cognition equation). In the case of our species, the speciation event associated with anatomical modernity (or perhaps even earlier events!) brought with it the genetically-endowed capacities required for behavioural modernity, which then slowly, in fits and starts, arose with the development of social environments scaffolding high-fidelity learning as well as sufficient demographic density to buffer information-flow and enable divided labor.

Thus, the discovery of various signals of behavioural modernity across African time and place, and of others associated with archaic hominins (both in Africa and in Eurasia), as well as new theoretical horizons, have significantly disrupted once-settled conceptions of how the transition could have occurred, and what the transition even consisted of.

\section{Rubicon expectations}

Caesar's crossing of the river Rubicon is framed as a kind of switch-point: a critical decision on his part which was necessary for the Roman Republic's fall and the rise of its Empire. Similarly, many investigations into behavioural modernity retain classificatory and explanatory practices focused on an isolated 'moment' where humans became human, or on a major proxy that should fully account for the transition. The shifts we've seen in our understanding of behavioural modernity undermine such approaches. We'll make our argument, before contextualizing it with respect to common archaeological practice.

\subsection{Against Rubicons}

We call "Rubicon expectation" in the context of behavioural modernity the practice of establishing a boundary that is expected to provide a non-ambiguous and universally applicable benchmark for modernity, and then monitoring the archaeological

\footnotetext{
${ }^{4}$ See for instance Klein's (2019) recent reconfirmation of the hypothesis of a new climate-driven gene constellation, affecting cognitive and communicative potential.
} 
record for it, thus inferring which hominin group, when and where, crossed it. Once such a benchmark for modernity is set, hominin populations/species are judged as "behaviourally modern" if their material culture meets it.

Three intertwined issues are at play that are worth distinguishing more clearly. One problem (i) involves the idea that the behavioural modernity phenomenon can be reduced to a major, big breakthrough, i.e. a key-trait model of what makes us humans different from other hominin lineages. ${ }^{5}$ A second, related problem (ii) concerns translating this view into non-ambiguous material signatures that can be identified in different archaeological contexts. A third (iii) involves interpreting the underlying processes that produced such signatures. As seen in the previous sections these interpretations have long had an inclination towards 'switch-point' explanations, often based on biologically mediated or hardwired causal factors. Our argument, in short, is that the new evidence and models we discussed in the previous section have transformed our conception of (iii), but many of the approaches underwriting (i) and (ii) have not as yet caught up.

As we saw in the previous sections, a number of empirical findings regarding the timing, location and pace of the appearance of innovations are no longer consistent with explanations attributing them to concomitant genetic or otherwise biologically mediated processes (iii). These have pushed research towards a picture in which the mosaic appearance and disappearance of innovations in both the African and the Eurasian record is explored through multiple, interacting factors-demographic, social, cultural transmission, environmental, ecological (cfr. d'Errico \& Banks, 2013)-before coalescing in a stabilized and expanding human niche (cfr. Foley, 2016; Kissel \& Fuentes, 2018; Sterelny, 2011). Despite this, Rubicon expectations and classificatory approaches (involving i, ii) are retained from older explanatory strategies, falling out of phase with respect to current theoretical and empirical advancements (Sect. 4 will develop a philosophical account of this in terms of 'investigative disintegration'). Rubicon expectations, we argue, might bias research in that they (still) implicitly depict the evolution of behavioural complexity as a demarcation problem, revolving around the identification of a major event, i.e. the onset of a competence or class of features capable of telling apart modern from non-modern records, human from non-human makers. By placing the explanatory and empirical burden on the Rubicon, distortions easily arise.

We've seen how the attempt to use the European UP material record as a Rubicon for other archaeological contexts to meet failed, most significantly for empirical reasons. However, pushing back the temporal horizon for the purported boundary or focusing on one major demarcation criterion for behavioural modernity, such as symbolically mediated behaviour treated as a "golden spike", does not escape the idea that behavioural modernity requires a significant Rubicon to be crossed. Let us briefly consider this last case and the problems it poses.

Symbolic behaviour and related inventories of symbolic expression are considered by many to be the crux of modernity (Chase \& Dibble, 1987; Henshilwood \& Marean, 2003; Marean, 2015; Tattersall, 2008; Wadley, 2001). Although what is classified as symbolic varies considerably (Kissel \& Fuentes, 2018), generally material items such

\footnotetext{
5 See Currie (2019a) for general discussion of such one-shot hypotheses.
} 
as personal ornamentations, use of ochre (with no discernible function), artwork, and practices like burial and other ritualistic activities, are linked to symbolic expression. However, artifacts and practices are not inherently imbued with symbolism and the lack of information about the cultural systems in which those artifacts acquired meaning poses a concrete limitation, making physical symbols equally fallible indicators of cultural richness (Sterelny, 2011, Kissel \& Fuentes, 2017, Currie and Meneganzin forthcoming).

Besides this, a more fundamental problem derives from what pushes the intuitive urge of identifying a Rubicon in the first place. That is, the belief that symbolic behaviour (or whatever else) captures the quintessence of being human, a trait (however complex) that should be considered exclusive to our lineage (White, 1940). In the case of symbolic expression, this seems evident in Henshilwood and Marean (2003) equating the expression "modern human behaviour" with "fully symbolic sapiens behaviour" (p. 644). This reproduces an old methodological problem. Replacing the old trait-list with an archaeological golden spike does no more than recreate the form of circular reasoning previously criticized regarding the UP 'revolution': if we define the capacity for symbolism as an exclusive human trait—being it, again, empirically derived from the material record of AMHs - we already know which assemblages will pass the modernity test.

Nonetheless, recent findings (although not without controversy) suggest that archaic populations were capable of symbolic expression, blurring the purported boundary between symbolic and non-symbolic species. Early glimmerings of possible instances of symbolic material culture (like abstract engravings and carvings) are reported at very ancient sites (800-300 kya, according to Colagè \& D'Errico, 2018), suggesting that the capacity for symbolic thought may have deeper phylogenetic roots. One ancient evidence of symbolic expression has been attested from an engraved clam shell from Trinil (Java), dated at ca. 540-430 kya and attributed to Homo erectus (Joordens et al., 2015). More significantly, different European Neanderthal sites contain traces that have been suggested to be symbolic in nature. These include burials, pigment use, personal ornamentations, collection of natural rare items and possibly cave art (Hoffman et al., 2018; Hovers \& Belfer-Cohen, 2006; Villa \& Roebroeks, 2014; Zilhao, 2007). When these expressions are not discarded as illegitimate or ambiguous in their intentions (probably because their evaluation is, in some cases, highly dependent upon the AMH-derived yardstick, i.e. the idea there is only one way of being truly symbolic), moving targets for behavioural modernity easily arise.

Strictly related to the issue of circularity is a second methodological problem, which we take to be even more pressing: capturing change in a mosaic-like transition. If the evolution of modern behaviour and complexity does not follow a unique path of development, but takes place along multiple trajectories, defined by different times, modes, locations and including various combinations of traits, then the processual nature of the phenomenon makes Rubicons unable to snap-shot a clear dichotomy between modern and non-modern assemblages, simply because no harsh dichotomy is to be expected. This also means that every temporal 'photograph' in the evolution of a complex feature such as symbolic expression must be interpreted from an evolutionary standpoint, i.e. bearing in mind that early glimmerings - or even evidence associated with other hominin groups - are expected to be different from 
Late Pleistocene or even modern-day manifestations. Thus, instead of seeing proxies of behavioural modernity as indicators of whether we have behavioural modernity or not—classifying the relevant population as 'archaic' or 'modern' - they are instead data relevant for understanding the mosaic of processes which eventually enabled cultural, informational and demographic stabilization.

This brings us to the explanatory dimension. No proxy in itself, not even symbolic expression, can alone have special significance. Placing the Rubicon when symbolic manifestations already show a stabilized signal (as Klein, 2019 seems to do in recognizing an abrupt inflection in the record, marked by the appearance of the LSA in Africa, and simultaneously, of the Eurasian Upper Palaeolithic) is not particularly informative nor decisive for assessing questions surrounding behavioural modernity, if it is not subsumed under the question of which mechanisms allow for the retention and proliferation of those expressions, as well as for the toleration of their costs. This does not mean downplaying the importance of detecting manifestations of symbolic expression (or other traits) in the archaeological record: rather, explanatory efforts should be directed at the mechanisms behind the transition, not its archaeological symptoms. As Ames et al. (2013) effectively put it:

Shifting the focus to identifying broader patterns of human adaptive strategies in social and ecological context still depends on our ability to document fluctuations in material culture through time and space, but it differs from the practice of monitoring the presence/absence of traits by singling out the range of behavioural strategies employed as the target of analysis, as opposed to trying to figure out whether or not a given assemblage passes the modernity threshold. In a way, this is an operationalization of the recognition that modern behaviour is a mosaic concept that needs to be interpreted as a function of its wider social and ecological contexts (Ibid. 36)

Thus, on our view, the continued use of Rubicons to demarcate behaviourally modern from non-modern hominins represents an outdated and misplaced expectation that is ill-fitting with respect to current knowledge and awareness of the complexity of the phenomenon. However, isn't it the case that linking material culture to social and cognitive capacities is common in archaeology? In the next subsection, we'll further contextualize our position.

\subsection{Archaeological business-as-usual and Rubicons}

Behavioural modernity, as we've seen so far, is identified in the record via archaeological assemblages taken to be signals of complex cognitive and social traits. This, in many ways, is archaeological business-as-usual. Archaeological categorization typically identifies cultural groupings via signals in the archaeological record: the 'Clovis peoples' identified by arrowheads across North America being a famous example. In paleoanthropological contexts, such categories are typically highly theoretical, based on ideas of the minimal cognitive, technological and social capacities required to construct such material remains (Currie \& Killin, 2019, Pain forthcoming). Such inferences often aim to identify the latest time that the capacity is present. So, hominin 
groups with, for instance, ochre use or beaded shells (keeping in mind the ambiguities of artifact significance that we mentioned) are categorized as behaviourally modern because it is taken that such material items signal symbolic expression. This approach has led to focus on a series of 'Rubicons' which various groups have taken to have crossed or failed to: archaeologists examine assemblages, identifying some groups as modern and other groups as not. Rubicons also set the significance of finds: discovering, say, ochre use in earlier times, or at wider ranges with respect to models' expectations, are treated as highly surprising and worthy of publication in prestigious journals. As such, Rubicons do a lot of work structuring archaeological investigation.

In light of the shifting research agenda for behavioural modernity, however, we think Rubicon expectations are unproductive and distortive lenses which actively undermine research. Recent models of the emergence of behavioural modernity understand it as a 'process' rather than an 'event' (Kissel \& Fuentes, 2018), or as 'threshold' in the bandwidth and fidelity of expertise flow (Sterelny, 2011). We could describe it as an 'emergent property' that belongs to a social group and which arises (and becomes visible) from the collaborative functioning of a system of interrelated factors (biological, cognitive, social-demographic, cultural). More specifically, we could envisage feedback systems of interactions according to which cultural practices can have direct effects on the cognitive capabilities of hominin populations and the neural substrates of individuals, constructing developmental environments to which future generations are exposed and amplifying learning capacities (see also Heyes, 2018).

Despite differences, recent approaches hold in common the idea that the material record does not simply track innate capacities-what Sterelny $(2011,2017)$ calls the 'simple reflection model' and whose 'bottom-up-only' direction of dependence is criticized by Colagè and d'Errico (i.e. the idea of a straightforward chain from genetic changes, to brain anatomy and physiology, to cognitive skills, to the package of cultural innovations that make us human, cfr. Colagè \& d'Errico, 2018). Rather, signals in the record showing the preservation and expansion of innovations reflect the culturally evolved and maintained epistemic niche of human groups. As such, inferring from the absence of material symbolism (say) to the fact that it did not exist and hence to archaic capacities - categorizing that group as non-behaviourally modern-is a mistake. It may rather reflect contingencies in that group's ecological and cultural context, not innate capacities. More careful use of proxies, such as simply being signals of the latest possible presence of the capacities (Killin \& Pain, 2021) are also undermined. On these models, we're not primarily interested in identifying when the innate capacities may have arose (as the basic prerequisites might have a deeper evolutionary history, and they're potentially spread across many hominin taxa), but in mosaic patterns of loss and stabilization.

Further, identifying particular finds as significant in virtue of, as it were, being in the wrong time and place is not particularly informative, nor does it meet the explanatory objectives of today's research. If we're looking for a Rubicon, then finds in earlier times or different locations are anomalous; however, if we're expecting a threshold effect they are not. 


\section{4. Disintegration}

It might be tempting to read the scientific developments thus far via hum-drum processes of hypothesis-testing. The hypothesis that behavioural modernity consists in changes in intrinsic cognitive capacities arising as the result of a genetically-mediated 'quantum leap' in a localized population and region (e.g. Bar-Yosef, 1998; Klein, 1989a, 1989b, 2000, 2013, 2019; Mellars \& Stringer, 1989) came under increasing empirical pressure as new paleoanthropological and archaeological data came online, leading to the development of new hypotheses which better accommodated the picture's complexity. There is no doubt something to this: such hypotheses have indeed been tested and put under pressure (cfr. Mallick et al., 2016); but in this section we'll argue for a different reading. The new data doesn't simply test hypotheses, they also transform our conception of target phenomena, the investigation has 'disintegrated', and this has important consequences for the kinds of explanations we should prefer and the epistemic structure of the investigation.

We'll foreshadow our account by responding to two immediate worries. First, you might think the term 'disintegration' is a bit dramatic: as we'll discuss below, we've not had to abandon swathes of empirical data relevant to behavioural modernity, and indeed in 5 we'll argue in defence of retaining the notion itself (once adequately updated). ${ }^{6}$ But disintegration comes in degrees. ${ }^{7}$ We've shifted from behavioural modernity being an integrated package of cognitive traits aligned with a particular set of assemblages, to something much more disparate and complex in its explanatory dimensions. It is this partial process that our account of disintegration seeks to capture.

Second, you might worry that the driving thought-that new conceptions of phenomena can change what counts as explanatorily adequate-is trivial. If we're convinced of the context-sensitivity of explanation, then of course changes to context involve changes in explanatory adequacy. But this is too quick: investigative disintegration involves more than changes to what explanation we want, it also involves changes to an investigation's epistemic structure, and this can have important consequences for, for example, the significance of new finds (we'll discuss some of these upshots in Sect. 5). Beyond explanation's context-sensitivity, we are interested in the dynamics of explanation, something Max Dresow has recently highlighted:

[the dynamics of explanation illuminates] the temporal dimension of explanation considered as an open-ended and collaborative process. Here we are not concerned with single explanations (or at least we needn't be), but rather with teams of explainers working jointly or in competition. (Dresow forthcoming, 2, italics in original)

As Dresow points out, the dynamics of explanation lead us to focus on the relationship between how explanations unfold and the investigative contexts in which they

\footnotetext{
6 We can indeed imagine more dramatic forms of disintegration, for instance cases in which it is no longer possible to save anything from the previous understanding of the target phenomenon (we would intuitively call such cases "disruption" or "dissolution" of the target phenomenon). But exploring them would bring us beyond the scope of the current paper.

7 It might help the reader to space the word out: dis-integration.
} 
are embedded. For behavioural modernity, new empirical findings and new theoretical tools have radically reshaped explanatory expectations and goals, which we'll characterize in terms of 'investigative disintegration': new understanding of the phenomenon, driven by new data and new interpretations of already available evidence, has reshaped the kind of questions we're asking and the kind of answers we want. Following Dresow, the dynamics of explanation are not simply about explanation, but about how explanatory expectations interact with, and are shaped by, efforts to characterize phenomena.

To capture the notion of investigative disintegration we'll turn to Alan Love's work.

\title{
4.1 Problem agendas
}

The notion of a problem agenda is useful for framing investigative disintegration. A 'problem agenda' is, in effect, a list of questions unified not by a theoretical perspective nor method, but by a phenomenon of interest. As Love puts it: "A problem agenda... is a "list" of interrelated questions (both empirical and conceptual) that are united by some connection to natural phenomena" (Love, 2008, p. 877). The agenda sets the role that various explanatory and evidential components play in understanding that phenomenon, particularly setting explanatory adequacy. That is, what kinds of answers count as a passable answer to the question at hand-if you want, which hypotheses are relevant. Further, this assigns 'explanatory burden' to some components of research over others.

\begin{abstract}
Because general criteria of explanatory adequacy provide structure to the explanatory burden associated with problem agendas, this epistemological account has the resources for ascertaining what disciplinary contributions are prerequisites for adequate explanations. The structure of a complex problem set derived from making the criteria of adequacy explicit highlights what different conceptual resources must be drawn upon to produce an acceptable explanatory vantage point (Ibid, 878).
\end{abstract}

Research into the deep past is by its nature multidisciplinary and integrative: the traces of past events and trends are often scarce, distorted and varied, requiring scientists to draw together a wide range of techniques, methods and theoretical resources to integrate the varied evidence available from historical records (Chapman \& Wylie, 2016; Currie, 2018). Problem agendas are a useful way of understanding the various roles data and theory can have in these complex investigations. To illustrate this through a nonhuman example, let's dip our toes into discussion of the evolution of obligate grazing amongst ungulates in the later Miocene (based on Janis, 2008).

The basal diet of ungulates is based on browsing, consuming the leaves and stems of plants, as well as fruit. Although grass arose earlier, it didn't become an extensive feature of global biota until the later Cenozoic (say, 25ma). As grass spread, so also did grazing in ungulates as they adapted to the new environments. Obligate grazing arose in Equids in North America and Bovidae in Africa. A relevant phenomenon of interest is variation in size between the obligate grazers, horses and bovids, and the browsers 
which includes giants such as rhinoceros, giraffe and elephant. In short, browsers are able to attain larger sizes than grazers: why?

Identifying phenomena like size differences between grazers and browsers generates problem agendas, which we can understand as setting the role various data and theory play in the investigation. First, some evidence, as it were, frames the investigation: data about the timing of grassland spread and of related radiations, as well as general comparisons between browsing and grazing strategies. Second, many threads play a role in characterizing the phenomenon at hand: establishing the uniqueness of bovids and equines involves phylogenetic analysis, establishing the difficulties of grass consumption and digestion, and the particular strategies adopted by various ungulates. Framing and characterizing the phenomenon help set explanatory adequacy but does not meet it. An explanatorily adequate answer to our question must identify what is different about obligate grazers in contrast to other ungulates such that they are limited in size. ${ }^{8}$

For instance, consider the role of highly hypsodont molars. Hypsodont teeth have high crowns and enamel reaching beyond the gum-line, and are commonly understood as an adaptation for dealing with abrasive vegetation. However, "not all hypsodont ungulates are grazers, as the silica contained in grass is far from the sole abrasive element in a herbivorous diet" (Janis, 2008, p. 29). Because hypsodoncy cannot tell between grazers and other ungulates, it alone doesn't meet explanatory adequacy-cannot carry the explanatory weight - of the problem agenda. However, it can play a role in framing and characterizing by helping identify mixed dietary and grazing strategies when they arise in the fossil record. What is needed for adequacy is identifying a relevant difference between the various lineages.

For instance, consider Clauss et al (2003)'s explanation. Obligate grazers typically use fore-gut digestion and are ruminants, an adaptation allowing for the digestion of grasses. Ruminants rely on delaying the passage of digestion, in order to have digesta available for re-chewing. Thus, digesta passage-time in ruminates is slower than in hindgut fermenters, and passage time scales with body-size. So, the larger a ruminant becomes, the longer digestion takes. This limits body-size because the efficiency by which acetic acid is converted to methane and carbon dioxide breaks down at longer digestion times. So, because hindgut fermenters generally have quicker digestion, they can afford larger sizes than foregut fermenters. Clauss et al.'s explanation is adequate due to identifying a relative difference between hindgut and foregut fermentation, capturing why the former have size constraints the latter do not.

Let's apply the example. We can understand a problem agenda as structuring the various roles data, evidence and theory plays in an investigation. An agenda picks out a particular phenomenon (that hindgut fermenters attain larger sizes than foregut ruminants) and in virtue of this some information takes on characterizing roles (size patterns and phylogenetics amongst ungulates), some framing roles (the various mechanisms and efficiency of digestive strategies) and others the explanatory burden set by explanatory adequacy (the constraints foregut digestion place on size). It doesn't follow from this that Clauss et al.'s explanation is right, simply that it is explanatorily adequate given the problem agenda.

\footnotetext{
8 This in part relies on contrastive accounts of explanation, see for example Lipton (1990).
} 
Problem agendas can be highly sensitive to both empirical context and explanatory interests. Switching our question, say, to the timing of the arisal and spread of obligate grazing, restructures the investigation: adequacy now calls for capturing why ten million years ago, and not earlier, ruminants arose. But this should be no surprise to philosophers used to the context-sensitivity of explanation. What we learn from Love's framework is that it is not simply explanatory adequacy that shifts with context, but the roles data and theory play. Further-and this is critical for investigative disintegration-learning new things about the underlying phenomenon can radically reshape explanatory adequacy and the agenda at large.

\subsection{Investigative disintegration}

As mentioned in the introduction, philosophers of science have rightly shifted from general accounts of unity and disunity to accounts of local integration and independence across several dimensions (data, evidence, hypotheses, institutions, etc....). Less attention has been paid to what happens when phenomena of study turn out to be far different from once thought; when investigations 'disintegrate', a process which has plausibly occurred for behavioural modernity. In this section, we can characterize one form of investigative disintegration by drawing on Love's machinery. In short, new information about the nature of the phenomenon ${ }^{9}$ - shifts in how it is characterized-can dramatically reshape investigation. This has consequences both for what constitutes an adequate explanation of behavioural modernity, and how we should read it from the archaeological record.

Let's remind ourselves, then, of how the phenomenon of behavioural modernity has changed, before characterizing the notion of disintegration abstractly.

We began tackling a relatively clear archaeological signal, the apparently sudden emergence, in the European UP, of a rich assemblage associated with symbolic expression, complex tool-use and so forth. This phenomenon shaped a problem agenda. First, the signal frames the investigation by discriminating between the biological emergence of $H$. sapiens and the appearance of behavioural modernity. Second, the distinction between anatomical and behavioural modernity sets relevant questions: what explains the ca. 150 kya gap between our species' evolution and our becoming behaviourally modern? Further, why was the emergence so sudden? Third, such questions drove a set of expectations: the explanatory weight is carried by a saltationist, often geneticallymediated mechanism involving a set of key innovations in a local population.

However, over twenty years anomalies arose which didn't simply undermine saltationist hypotheses, but reshaped the phenomenon, problem agendas and standards for explanatory adequacy. The discovery of increasingly diffuse, often partial, aspects of the assemblage identified with behavioural modernity earlier in the record challenges a saltationist picture and the idea that we should explain the origins of a 'syndrome', but so too does it challenge the usual way of understanding the distinction between behavioural and anatomical modernity. Instead of marking a genetic transition, we

\footnotetext{
9 By 'phenomenon' we mean something similar to Bogen \& Woodward (1988): a recurrent pattern inferred from data.
} 
instead see a mosaic of potential precursors. Explanatory adequacy shifts from requiring a story about a single, localized origin, to asking after stabilization. Models of learning, social organization, demographics and niche stabilization take on explanatory weight previously carried, under many instances of "revolution" scenarios, by genetic or biological evolution. Again, as seen in the discussion of the evolution of obligate grazing, it doesn't follow from this that such specific models are inevitably right, but that they are explanatorily adequate. ${ }^{10}$ On this basis we can characterize investigative disintegration abstractly:

Investigative disintegration, in such instances, occurs when: (1) the characterization of a phenomenon changes sufficiently for (2) different components of the problem agenda to take on explanatory weight. ${ }^{11}$

Note that our definition of investigative disintegration doesn't specify which factors might change how phenomena are characterized: we suspect a wide variety of influences could be at play, and want to avoid overgeneralizing from our example. For it is likely idiosyncratic. Investigation of behavioural modernity operates under 'epistemic scarcity' (Currie forthcoming): like many studies of the deep past, data is rare, fragile and difficult to manage. In virtue of this, phenomena are often highly sensitive to new incoming data. This scarcity in part explains how expanding paleoanthropological and archaeological discoveries (along with new theoretical frameworks) could so thoroughly reshape the epistemic landscape. Investigations with more systematic and manageable data could prove more intransigent.

Moreover, we're concerned here with forms of disintegration involving shifts in phenomena. Specifically, new ways of characterizing the phenomenon, driven by new finds and theoretical innovation, led to very different requirements in explanatory adequacy. But this is likely but one way the dynamics of investigation might shift. Other examples may include cases where changes in theory play a central role, say, but we'll leave that more expansive discussion for later work.

There are similarities between the phenomenon we highlight here and older systems within the philosophy of science. For instance, new evidence pertaining to behavioural modernity could be aligned with Kuhn's 'anomalies' (Kuhn, 1962), and the shifts in our conception of behavioural modernity might be aligned with shifts in Lakatosian 'cores' or progressive or regressive problem shifts (Lakatos, 1976). It may be that what we pick out here could be captured with this machinery. However, there are important differences: both Kuhn and Lakatos are significantly more systematic than we wish to be. For instance, comparing a Kuhnian 'paradigm shift' to investigative disintegration reveals significant differences between them. We'll briefly highlight two.

First, differences in the degree of disintegration: because paradigms holistically structure research programs they appear to be an all-or-nothing affair, while the finergrained structure afforded by disintegration can better capture the actual dynamics

\footnotetext{
10 Note also how this differs from a simple case of epistemic pluralism. For pluralism to occur, under this perspective, multiple accounts must equally satisfy criteria of explanatory adequacy. But this is not the case of old and more recent Rubicon-based approaches, as seen above. There is however room for much pluralism regarding the nature of the stabilizing processes underwriting modernity.

11 It might be tempting to see this as the arisal of a new problem-agenda. We don't think much hangs on how precisely we delineate problem-agendas, context-sensitive as they are.
} 
of research. This affords side-stepping long-toothed worries about incommensurability while nonetheless allowing critical engagement with the dynamics of research. Although changes in how behavioural modernity is conceived has not led to a breakdown of communication or method, we've argued that it has involved certain practices-particularly those related to the categorization and significance of new finds-dragging behind the epistemic purposes of the reforming agenda.

Second, the scope and organizing principles of research agendas differ from paradigms. It isn't clear that behavioural modernity is anything like a paradigm (or the core of a research program for that matter): problem agendas tend to come into being in piecemeal, messy ways and tend to focus on significantly narrower targets than paradigms. More importantly, as research agendas focus on phenomena and how scientists characterize these, they are more appropriate for behavioural modernity than more 'theory-centric' approaches. This is simply because in this instance it has not been changes to wide-scale theory, nor evidence undermining such theory, that has ignited the change: it is primarily how new finds (and the reappraisal of old ones) have reshaped how the phenomenon is characterized.

Having said this, we are open to understanding these notions as ways of (as an anonymous reviewer nicely put it) 'rescuing the morals of Kuhn' ${ }^{12}$ In a sense at least, understanding investigative disintegration is in step with understanding anomalies and their subsequent revolutions insofar as both are fundamentally interested in how unexpected discoveries shape the dynamics of research. The crucial difference, we think, is that investigative disintegration follows the crucially local, practice-oriented and dynamic turn of recent philosophy of science (a turn which, we've briefly suggested, is for the better, at least in this context).

There is a certain tension in our use of the term 'disintegration': after all, it was the integration of new data which proved so thoroughly transformative. But this is what we should expect. Integration at some level can lead to disintegration at another, as science's epistemic tapestry is warped around new discoveries, analytic techniques and models. This suggests a positive epistemic role for disintegration: too-closely integrated research programs might well lead to a too-strict, restricted, investigation. Such investigations are likely blind to alternative hypotheses due to a too-conservative focus (Currie, 2019b; Stanford, 2006, 2019, Schneider forthcoming).

Our account then provides an additional nail in the coffin of the "jigsaw puzzlemetaphor", often invoked to (mis)represent archaeological practice (see Chapman \& Wylie, 2016). The metaphor involves conceiving research progress as filling gaps and finding new pieces of information that will fit pre-existing schemas. Rather, empirical findings concerning behavioural modernity have, in an iterative fashion and with much theoretical innovation, led to the re-conception of what the phenomenon itself is, how it should be categorized, and how we should explain it.

We don't wish to derive more general normative claims about the role of disintegration in the dynamics of science, as there well may be more disruptive cases where target phenomena are completely dissolved and research achievements are not preserved (these may be instances analogous to Kuhn-loss, see also footnote 6; in the

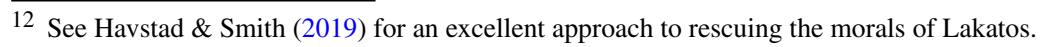


following section we'll explain why this is not so for our case). Instead, in less dramatic circumstances, via 'shaking up' the research program, disintegration can lead to bursts of new ideas: something we're witnessing for behavioural modernity. Thus, our position is not merely a description of the dynamics of research, there is also scope for understanding investigative disintegration as progressive, at least in the instance we've applied it to.

\section{The fate of 'behavioural modernity'}

In light of its mosaic emergence, the disparateness of its proxies and troublesome baggage both from Eurocentric origins and the hunt for Rubicons, we might be tempted to abandon 'behavioural modernity' altogether. Shea (2011), for instance, attacks it for being qualitative, essentialist, and replicating the binary dichotomy between modern and nonmodern states. However, we think the abandonment of the notion should be resisted for three reasons.

First, Shea proposes to focus instead on 'behavioural variability', defined as a "measurable quality of all human behaviour expressed in terms of modality, variance, skew, and other quantitative/statistical properties" (ivi, p. 2). However, it is not clear how a focus on behavioural variability would avoid recreating old operational problems in a different context: to measure variability, some units of input will have to be counted and what units to consider will need to be decided, thus risking to reintroduce the flaws of the old dreaded trait list approach and research agenda.

Second, even after rejecting the problematic legacy of behavioural modernity, there is still something to be explained, making the adoption of a new notion too quick a move (perhaps even unnecessary). Shea seems to treat the idea that a relevant transition might have occurred as illegitimate (as reflected in the title of his paper, "Homo sapiens is as Homo sapiens was"). The archaeological assemblages associated with hominin activity 300-200 kya display a radically different pattern of cultural and ecological flexibility, complexity and density to those 100-50 kya. Explaining 'behavioural modernity' is to explain those genuinely puzzling differences in the record, despite the radical changes in how they are now conceptualized.

Third, there is a potential unity to the explanations which are emerging, as indeed we might expect from some cases of investigative disintegration. That unity is not found in any pre-defined cultural expression or assemblage. There is not necessarily a particular signature or set thereof, be they 'golden spikes' or a trait-list, associated with behavioural modernity. But contingency and diversity at the level of cultural assemblage doesn't undermine another kind of unity.

As we've seen, contemporary explanations point to demographic scale and sociocultural innovations (such as high-fidelity and high-volume social learning) as being necessary for the expression and stabilization of diverse hominin expressions. If they continue to bear out empirically, we should conclude that it is those processes which explain (or perhaps even constitute) behavioural modernity. The new explanations, then, are unified via pointing to a set of cultural and demographic processes regardless of the particular cultural and technological expressions they underwrite, the taxonomic affiliations at hand, or the material records that signal them. 
This leads us to two final points. First, we've argued that Rubicon practices and expectations in classification (classifying assemblages as 'modern' or 'archaic', for instance) are wrong-headed. This is due to the progressive disintegration of the problem agenda-from conceiving of behavioural modernity as a shift in innate capacities to a shift in epistemic niche-and the complex nature of the target phenomenon, that Rubicons (otherwise not infrequent in archaeological investigations) are inappropriate for this kind of study.

So, what should we do instead? Our argument in no way implies that discoveries in fieldwork are unimportant for filling out our picture of the emergence of behavioural modernity. Rather, it suggests such discoveries play a different role than previously thought. New finds do not shift when purported Rubicons were crossed, but instead help test and enrich models of how the mosaic emergence of behavioural modernity occurred and which conditions allowed for such threshold effects to become visible. As we've said, categorization in paleoanthropology is not theoretically innocent, but rather reflects the presumed mechanisms of evolutionary and cultural change. As we underlined above, finds and assemblages should be interrogated with respect to how they document cultural changes and the underlying mechanisms at a regional scale, not as reflecting shifts in in-built capacities.

Second, Rubicon-based practices often govern what counts as significant in archaeology: it is discoveries which expand Rubicons that deserve special place in high-profile prestige journals, for instance. Finds which are earlier than we expect, or from unexpected geographical regions, take on the most significance as they shift "implicit" Rubicons or encourage revising outdated assumptions. Even within the processual understanding of behavioural modernity, finding precursors may be surprising: discovering, say, a flourishing musical tradition complete with tuned instruments $150 \mathrm{kya}$ would be quite remarkable. But if we think behavioural modernity is a mosaic and eventually stabilizing process reliant on demographic and cultural innovations, earlier precursors become less anomalous and their significance should be treated differently. Ultimately what matters is enriching our picture of how those processes were enacted across different contexts.

\section{Conclusion}

We've aimed to both provide an analysis of how investigations of behavioural modernity have been reshaped over thirty years, and cover a hitherto underexplored feature of the dynamics of scientific research. Regarding the latter, where philosophers have analysed how new research agendas and repertoires form and stabilize, we've considered processes of disintegration: how evolving conceptions of phenomena can reshape what counts as explanatorily adequate for a research agenda. Regarding the former, behavioural modernity's mosaic, partial and incremental nature undermines the still-common archaeological practice of categorizing assemblages and complex, transitional phenomena via strict Rubicons.

During periods of destabilization and disintegration various scientific practices shift at different rates. We have analysed a disintegration process that began decades ago and it is still ongoing, with some practices not being aligned with the current problem 
agenda. We have also suggested - although we leave a more thorough examination to a future project - that investigative disintegration, in the form we analysed (as shifts in target phenomena), is a phase in the dynamics of research that can play a positive epistemic role, fostering new conceptual reorientations and eventually new forms of unity to explanations.

There is much more to be said about investigative disintegration. As we've suggested, our example is likely idiosyncratic: examination of other cases is likely to demonstrate different dynamics and may be less focused on shifts in phenomena. Further, we've said nothing about the role of disciplinary and institutional shifts in disintegration, nor about broader social factors in driving scientific change. As for behavioural modernity, although the concept should be understood as a threshold phenomenon rather than a discrete revolution, the notion still plays a critical role in holding together research agendas surrounding the evolution of our species.

On our view, the new picture emerging of the evolution of $H$. sapiens has already put in place ways of rethinking the purpose and practice of how we identify and conceptualize behavioural modernity in the first place - a picture that points towards a complex and contextualized interplay between demographic and cultural factors, as well as ecological and biological dimensions, that are responsible for the stabilization and flourishing of 'modern' human lifeways and diversity.

Acknowledgements This paper was read by Exeter University's students of the "Deep Past, History and Humanity" course (Lead tutor: A.C.) and by the Egenis Research Exchange, who provided invaluable feedback. We are also grateful to Kim Sterelny and Luca Pagani for their useful comments and suggestions on drafts. We would also like to thank two anonymous reviewers, whose suggestions improved the quality and clarity of this paper.

Open Access This article is licensed under a Creative Commons Attribution 4.0 International License, which permits use, sharing, adaptation, distribution and reproduction in any medium or format, as long as you give appropriate credit to the original author(s) and the source, provide a link to the Creative Commons licence, and indicate if changes were made. The images or other third party material in this article are included in the article's Creative Commons licence, unless indicated otherwise in a credit line to the material. If material is not included in the article's Creative Commons licence and your intended use is not permitted by statutory regulation or exceeds the permitted use, you will need to obtain permission directly from the copyright holder. To view a copy of this licence, visit http://creativecommons.org/licenses/ by/4.0/.

\section{References}

Ankeny, R. A., \& Leonelli, S. (2016). Repertoires: A post-Kuhnian perspective on scientific change and collaborative research. Studies in History and Philosophy of Science Part A, 60, 18-28.

Ames, C. J., Riel-Salvatore, J., \& Collins, B. R. (2013). Why we need an alternative approach to the study of modern human behaviour. Canadian Journal of Archaeology/Journal Canadien d'Archéologie, 21-47.

Bar-Yosef, O. (1998). On the nature of transitions: The Middle to upper palaeolithic and the neolithic revolution. Cambridge Archaeological Journal, 8, 141-163.

Bar-Yosef, O. (2002). The upper paleolithic revolution. Annual Review of Anthropology, 31(1), 363-393.

Barham, L., \& Mitchell, P. (2008). The first Africans: African archaeology from the earliest tool makers to most recent foragers. Cambridge University Press.

Bogen, J., \& Woodward, J. (1988). Saving the Phenomena. The Philosophical Review, 97(3), 303-352.

Brigandt, I. (2010). Beyond reduction and pluralism: Toward an epistemology of explanatory integration in biology. Erkenntnis, 73(3), 295-311. 
Chang, H. (2012). Is water H2O?: Evidence, realism and pluralism (Vol. 293). Springer.

Chapman, R., \& Wylie, A. (2016). Evidential reasoning in archaeology. Bloomsbury Publishing.

Chase, P. G., \& Dibble, H. L. (1987). Middle palaeolithic symbolism: A review of current evidence and interpretations. Journal of Anthropological Archaeology, 6, 263-296.

Clauss, M., Frey, R., Kiefer, B., et al. (2003). The maximum attainable body size of herbivorous mammals: Morphophysiological constraints on foregut, and adaptations of hindgut fermenters. Oecologia, $136(1), 14-27$.

Colagè, I., \& d'Errico, F. (2018). Culture: The driving force of human cognition. Topics in Cognitive Science, 12(2), 654-672.

Conard, N. J. (2008). A critical view of the evidence for a southern African origin of behavioural modernity. South African Archaeological Society Goodwin Series, 10, 175-179.

Currie, A., \& Killin, A. (2019). From things to thinking: Cognitive archaeology. Mind \& Language, 34(2), $263-279$.

Currie, A., \& Meneganzin, A. (forthcoming). Hawkes' Ladder, Underdetermination \& the Mind's Capacities. In: Wynn, T., Overmann, K. and Coolidge, F. (eds.). Oxford Handbook of Cognitive Archaeology.

Currie, A. (forthcoming). Stepping Forwards by Looking Back: Underdetermination, Epistemic Scarcity \& Legacy Data. Perspectives on Science.

Currie, A. (2019a). Simplicity, one-shot hypotheses and paleobiological explanation. History and Philosophy of the Life Sciences, 41(1), 1-24.

Currie, A. (2019b). Existential risk, creativity \& well-adapted science. Studies in History and Philosophy of Science Part A, 76, 39-48.

Currie, A. (2018). Rock, bone, and ruin: An optimist's guide to the historical sciences. MIT Press.

Davies, S. (2019). Behavioral modernity in retrospect. Topoi, 40, 221-232.

Deacon, H. J. (2001). Modern human emergence: An African archaeological perspective. In P. V. Tobias, M. A. Raath, J. Moggi-Cecchi, \& G. A. Doyle (Eds.), Humanity from African naissance to coming millennia: Colloquia in human biology and palaeoanthropology (pp. 217-226). University of Florence Press.

D'Errico, F. (2003). The invisible frontier. A multiple species model for the origin of behavioral modernity. Evolutionary Anthropology, 12, 188-202.

D'Errico, F., Vanhaeren, M., Barton, N., et al. (2009). Additional evidence on the use of personal ornaments in the Middle Paleolithic of North Africa. Proceedings of the National Academy of Sciences, 106, $16051-16056$.

D'Errico, F., \& Henshilwood, C. S. (2011). The origin of symbolically mediated behaviour: From antagonistic scenarios to a unified research strategy. In C. S. Henshilwood \& F. D'Errico (Eds.), Homo symbolicus: The dawn of language, imagination and spirituality (pp. 49-73). Amsterdam: John Benjamins.

D’Errico, F., \& Stringer, C. B. (2011). Evolution, revolution or saltation scenario for the emergence of modern cultures? Philosophical Transactions of the Royal Society b: Biological Sciences, 366(1567), 1060-1069.

D’Errico, F., \& Banks, W. (2013). Identifying mechanisms behind middle paleolithic and middle stone age cultural trajectories. Current Anthropology, 54, S371-S387.

Diamond, J. (1992). The third chimpanzee. Harper Collins.

Dresow, M. (forthcoming). Explaining the apocalypse: the end-Permian mass extinction and the dynamics of explanation in geohistory. Synthese, 1-34.

Hovers, E., \& Belfer-Cohen, A. (2006). 'Now you see it, now you don't'-modern human behavior in the Middle Paleolithic. In E. Hovers \& S. L. Kuhn (Eds.), Transitions before the transition: Evolution and stability in the middle paleolithic and middle stone age (pp. 295-304). Springer.

Foley, R. A. (2016). Mosaic evolution and the pattern of transitions in the hominin lineage. Philosophical Transactions of the Royal Society b: Biological Sciences, 371(1698), 1-14.

Galway-Witham, J., Cole, J., \& Stringer, C. (2019). Aspects of human physical and behavioural evolution during the last 1 million years. Journal of Quaternary Science, 34(6), 355-378.

Gamble, C. (1994). Time walkers: The prehistory of global colonization. Harvard University Press.

Gamble, C. (1999). The palaeolithic societies of Europe. Cambridge University Press.

Havstad, J. C., \& Smith, N. A. (2019). Fossils with feathers and philosophy of science. Systematic Biology, 68(5), 840-851.

Henn, B. M., Cavalli-Sforza, L. L., \& Feldman, M. W. (2012). The great human expansion. Proceedings of the National Academy of Sciences, 109(44), 17758-17764. 
Henshilwood, C. S., \& Marean, C. W. (2003). The origin of modern human behavior: Critique of the models and their test implications. Current Anthropology, 44, 627-651.

Henshilwood, C. S., \& Dubreuil, B. (2011). The still Bay and Howiesons Poort, 77-59 ka: Symbolic material culture and the evolution of the mind during the African Middle Stone Age. Current Anthropology, 52(3), 361-400.

Heyes, C. (2018). Cognitive gadgets: The cultural evolution of thinking. Harvard University Press.

Hoffmann, D. L., Standish, C. D., et al. (2018). U-Th dating of carbonate crusts reveals Neandertal origin of Iberian cave art. Science, 359, 912-915.

Hublin, J. J., \& Ben-Ncer, A. (2017). New fossils from Jebel Irhoud, Morocco and the pan African origin of Homo sapiens. Nature, 546, 289-292.

Janis, C. (2008). An evolutionary history of browsing and grazing ungulates. In The ecology of browsing and grazing (pp. 21-45). Springer, Berlin, Heidelberg.

Joordens, J. C. A., D’Errico, F., Wesselingh, F. P., et al. (2015). Homo erectus at Trinil in Java used shells for tool production and engraving. Nature, 518, 228-231.

Killin, A., \& Pain, R. (2021). Cognitive archaeology and the minimum necessary competence problem. Biological Theory. https://doi.org/10.1007/s13752-021-00378-7

Kissel, M., \& Fuentes, A. (2017). Semiosis in the pleistocene. Cambridge Archaeological Journal, 27(3), 397-412.

Kissel, M., \& Fuentes, A. (2018). 'Behavioral modernity' as a process, not an event, in the human niche. Time and Mind, 11(2), 163-183.

Kissel, M., \& Fuentes, A. (2021). The ripples of modernity: How we can extend paleoanthropology with the extended evolutionary synthesis. Evolutionary Anthropology: Issues, News, and Reviews, 30(1), $84-98$.

Klein, R. (1989a). The human career: Human biological and cultural origins. The University of Chicago Press.

Klein, R. (1989b). Biological and behavioral perspectives on modern human origins in southern Africa. In P. Mellars \& C. B. Stringer (Eds.), The human revolution: behavioral and biological perspectives on the origins of modern humans (pp. 529-546). Edinburgh: Edinburgh University Press.

Klein, R. (2000). Archeology and the evolution of human behavior. Evolutionary Anthropology, 9(1), 17-36.

Klein, R. (2013). Modern human origins. General Anthropology, 20, 1-4.

Klein, R. (2019). Population structure and the evolution of Homo sapiens in Africa. Evolutionary Anthropology: Issues, News, and Reviews, 28(4), 179-188.

Kuhn, T. S. (1962). 2012. The structure of scientific revolutions. University of Chicago press.

Lakatos, I. (1976). Falsification and the methodology of scientific research programmes. In Can theories be refuted? (pp. 205-259). Springer, Dordrecht.

Lipson, M., Ribot, I., Mallick, S., et al. (2020). Ancient West African foragers in the context of African population history. Nature, 577(7792), 665-670.

Lipton, P. (1990). Contrastive explanation. Royal Institute of Philosophy Supplement, 27, 247-266.

Love, A. C. (2008). Explaining evolutionary innovations and novelties: Criteria of explanatory adequacy and epistemological prerequisites. Philosophy of Science, 75(5), 874-886.

Machamer, P., Darden, L., \& Craver, C. F. (2000). Thinking about mechanisms. Philosophy of Science, 67(1), 1-25.

Mallick, S., Li, H., Lipson, M., et al. (2016). The Simons genome diversity project: 300 genomes from 142 diverse populations. Nature, 538(7624), 201-206.

Marean, C. W., Bar-Matthews, M., Bernatchez, J., et al. (2007). Early human use of marine resources and pigment in South Africa during the Middle Pleistocene. Nature, 449, 905-908.

Marean, C. W. (2015). An evolutionary anthropological perspective on modern human origins. Annual Review of Anthropology, 44, 533-556.

McBrearty, S., \& Brooks, A. S. (2000). The revolution that wasn't: A new interpretation of the origin of modern humans. Journal of Human Evolution, 39, 453-463.

McDougall, I., Brown, F. H., \& Fleagle, J. G. (2005). Stratigraphic placement and age of modern humans from Kibish, Ethiopia. Nature, 433, 733-736.

Mellars, P. A., \& Stringer, C. B. (Eds.). (1989). The human revolution: Behavioral and biological perspectives on the origins of modern humans. Edinburgh University Press.

Mellars, P. (1989). Major issues in the emergence of modern humans. Current Anthropology, 30(3), 349-385.

Mellars, P. A. (2005). The impossible coincidence. A single-species model for the origins of modern human behavior in Europe. Evolutionary Anthropology, 14, 12-27. 
Mellars, P. (2006). Why did modern human populations disperse from Africa ca 60,000 years ago? A new model. Proceedings of the National Academy of Sciences of the United States of America, 103, 9381-9386.

Mitchell, S. D. (2003). Biological complexity and integrative pluralism. Cambridge University Press.

Mithen, S. (1998). A creative explosion? Theory of mind, language, and the disembodied mind of the Upper Paleolithic. In S. Mithen (Ed.), Creativity in human evolution and prehistory. New York: Routledge.

Nowell, A. (2010). Defining behavioral modernity in the context of Neandertal and anatomically modern human populations. Annual Review of Anthropology, 39, 437-452.

Pain, R. (2019). What can the lithic record tell us about the evolution of hominin cognition? Topoi, 40, 245-259.

Parravicini, A., \& Pievani, T. (2019). Mosaic evolution in hominin phylogeny: Meanings, implications, and explanations. Journal of Anthropological Sciences, 97, 1-24.

Potochnik, A. (2010). Explanatory independence and epistemic interdependence: A case study of the optimality approach. The British Journal for the Philosophy of Science, 61(1), 213-233.

Powell, A., Shennan, S., \& Thomas, M. (2009). Late Pleistocene demography and the appearance of modern human behavior. Science, 324, 1298-1301.

Renfrew, C. (1996). The sapient behaviour paradox: How to test for potential? In P. Mellars \& K. Gibson (Eds.), Modelling the early human mind (pp. 11-15). McDonald Institute.

Renfrew, C. (2009). Situating the creative explosion: Universal or local? In C. Renfrew \& I. Morley (Eds.), Becoming human: Innovation in prehistoric material and spiritual culture (pp. 74-92). Cambridge University Press.

Richerson, P., Robert, B., \& Bettinger, R. (2009). Cultural innovations and demographic change. Human Biology, 81, 211-235.

Schlebusch, C. M., Malmström, H., Günther, T., et al. (2017). Southern African ancient genomes estimate modern human divergence to 350,000 to 260,000 years ago. Science, 358(6363), 652-655.

Schneider, Mike D. (forthcoming) Creativity in the social epistemology of science. Philosophy of Science.

Shea, J. (2011). Homo sapiens Is as Homo sapiens Was. Behavioral Variability versus "Behavioral Modernity" in Paleolithic Archaeology. Current Anthropology, 52, 1-35.

Shennan, S. (2001). Demography and cultural innovation: A model and its implications for the emergence of modern human culture. Cambridge Archaeological Journal, 11, 5-16.

Stanford, P. K. (2006). Exceeding our grasp: Science, history, and the problem of unconceived alternatives (Vol. 1). Oxford: Oxford University Press.

Stanford, P. K. (2019). Unconceived alternatives and conservatism in science: The impact of professionalization, peer-review, and Big Science. Synthese, 196(10), 3915-3932.

Sterelny, K. (2011). From hominins to humans: How sapiens became behaviourally modern. Philosophical Transactions of the Royal Society B, 366, 809-822.

Sterelny, K. (2017). Artifacts, symbols, thoughts. Biological Theory, 12(4), 236-247.

Tattersall, I. (2008). An evolutionary framework for the acquisition of symbolic cognition by Homo sapiens. Comparative Cognition \& Behavior Reviews, 3, 99-114.

Tattersall, I. (2017). How can we detect when language emerged? Psychonomic Bulletin \& Review, 24(1), 64-67.

Villa, P., \& Roebroeks, W. (2014). Neandertal demise: an archaeological analysis of the modern human superiority complex. PLoS ONE, 9(4), e96424.

Wadley, L. (2001). What is cultural modernity? A general view and a South African perspective from Rose Cottage Cave. Cambridge Archaeological Journal, 11(2), 201.

Wadley, L. (2015). Those marvellous millennia: The Middle Stone Age of southern Africa. Azania: Archaeological Research in Africa, 50(2), 155-226.

White, L. (1940). The symbol: The origin and basis of human behavior. Philosophy of Science, 7(4), $451-463$.

Wylie, A. (1999). Rethinking unity as a "working hypothesis" for philosophy of science: How archaeologists exploit the disunities of science. Perspectives on Science, 7(3), 293-317.

Wynn, T., Overmann, K. A., \& Coolidge, F. L. (2016). The false dichotomy: A refutation of the Neandertal indistinguishability claim. Journal of Anthropological Sciences, 94, 1-22.

Zilhão, J. D. (2006). Neandertals and moderns mixed, and it matters. Evolutionary Anthropology, 15, $183-195$.

Zilhão, J. D. (2007). The emergence of ornaments and art: An archaeological perspective on the origins of "behavioural modernity." Journal of Archaeological Research, 15, 1-54. 
Publisher's Note Springer Nature remains neutral with regard to jurisdictional claims in published maps and institutional affiliations. 